\title{
Neue Wege in der dermatologischen Weiterbildung Teil 2: Klinik trifft Kunst: Sehschule für Dermatologen - die Ärzte der Hautklinik im Wilhelm-Hack-Museum Ludwigshafen
}

\author{
New Paths in Dermatologic Education \\ Part 2: Hospital Meets Art: Vision-Training for Dermatologists - Physicians of the Skin Hospital Visit the \\ Wilhelm-Hack-Museum in Ludwigshafen
}

Autoren

Institute

\section{Löser ${ }^{1}$, J. E. Weiss ${ }^{2}$}

Hautklinik, Hauttumorzentrum Rheinpfalz, Klinikum Ludwigshafen

vormals Wilhelm-Hack-Museum, Ludwigshafen/aktuell Zentrum für Literatur- und Kulturforschung, Berlin

\section{Bibliografie}

Dol http://dx.doi.org/ $10.1055 / \mathrm{s}-0030-1256637$

Online-Publikation: 28. 7. 2011

Akt Dermatol 2011; 37:

309-311 @ Georg Thieme

Verlag KG Stuttgart · New York ISSN 0340-2541

\section{Korrespondenzadresse} Dr. med. Christoph Löser Leitender Oberarzt der Hautklinik/Hauttumorzentrum Rheinpfalz

Klinikum Ludwigshafen

Bremserstraße 79

67063 Ludwigshafen

loeserc@klilu.de

\section{Zusammenfassung \\ $\nabla$}

Die Dermatologie stellt an den Facharzt den hohen Anspruch, Strukturen und Zusammenhänge wahrzunehmen, die auf der Haut jeder sehen kann, aber nur das geschulte Auge erkennt. Vor diesem Hintergrund wurden an der Hautklinik Ludwigshafen im Jahr 2010 neben den zahlreichen fachbezogenen Fortbildungen zwei Veranstaltungen durchgeführt, welche die Sensorik auf unkonventionelle Weise fördern sollten. Da visu-

\section{Betrachtung von Oberflächen \\ $\nabla$}

Das Auge sieht, was es sucht.

Max Slevogt

Medizinern, und Dermatologen im Besonderen, wird eine Affinität zur Kunst nachgesagt und Studenten profitieren in ihrer Ausbildung nachweislich von einer gezielten Exposition [1,4,5,6].

Was können Dermatologen im Museum lernen? Die Basis für die Diagnose in der Dermatologie ist die Betrachtung der Haut des erkrankten Patienten. Ähnlich verhält es sich bei einem Ausstellungsbesuch. Ein Exponat im Museum ist stets mit Tabus belegt: Das ausgestellte Kunstwerk darf in aller Regel nicht berührt oder ertastet werden - nur die intensive Betrachtung des Werks, seiner Außenhaut gleichsam, lässt die formale und inhaltliche Dimension erschließen. Nähe und Distanz zum Kunstwerk, Beleuchtung und Blickwinkel formen Oberflächen visuell. Was sich bei der Betrachtung aus großer Distanz als flächig und als Gestaltung in malerischer Perfektion darstellt, beginnt sich bei der Anschauung vis à vis mit Verläufen, Pfützenbildungen, Rissen, Wellen, Schichten, Eintrübungen und Beulen zu füllen. Die persönliche Handschrift des Künstlers wird plötzlich sichtbar und gibt dem Gemälde mit gro- elle Aspekte im Rahmen der hautärztlichen Diagnostik heute glücklicherweise überwiegen, fand die zweite Sonderveranstaltung für die Ärzte der Hautklinik im Wilhelm-Hack-Museum in Ludwigshafen statt. Unter dem Thema „Sehschule für Dermatologen“ führte Dr. Judith Elisabeth Weiss, seinerzeit Kuratorin des Museums, durch die Sammlung, die bedeutende Werke der Kunst des 20. Jahrhunderts unter dem Titel „hackordnung“ jährlich neu präsentiert.

ben, feinen, gestischen oder sensiblen Pinseldukten sein unverwechselbares Gesicht. In ganz besonderer Weise wird das Auge in einem der radikalsten Werkkomplexe der Kunst des 20. Jahrhunderts im Umfeld der Künstlergruppe Zero sensibilisiert, die im Wilhelm-Hack-Museum unter dem Motto „Weiße Stille“ wichtige Arbeiten versammelt. Im Streben nach Stille und Balance bieten sich weiße Leinwände dem Blick dar, die in der völligen Entleerung des Bildraumes nicht nur vom abbildbaren Gegenstand sondern auch von Farbe mit Leichtigkeit und Transparenz spielen. Wie feinste Gewebe offenbaren sich rhythmische Strukturen in weißen Rastern auf weißem Grund nebst ihren Schattierungen. Als „Augenerlebnisse" bezeichnete der führende Vertreter der Op-Art, Victor Vasarely, die Wirkung seiner Kunst. Farbliche Variationen geometrischer Formen wie Quadrate, Rauten, Dreiecke oder Stabformen sollten mit ihren optischen und virtuell-kinetischen Effekten die Netzhaut in Schwingung versetzen. Diese künstlerische Richtung der 1960er-Jahre mit ihren präzisen abstrakten Formmustern und geometrischen Farbfiguren erzeugen beim Betrachter überraschende Effekte, die von der Imagination von Bewegung über Flimmereffekte bis hin zu optischen Täuschungen reichen. Eine Erweiterung des Sehens war damit intendiert. Besonders die beiden Werke des Op-Art-Künstlers 


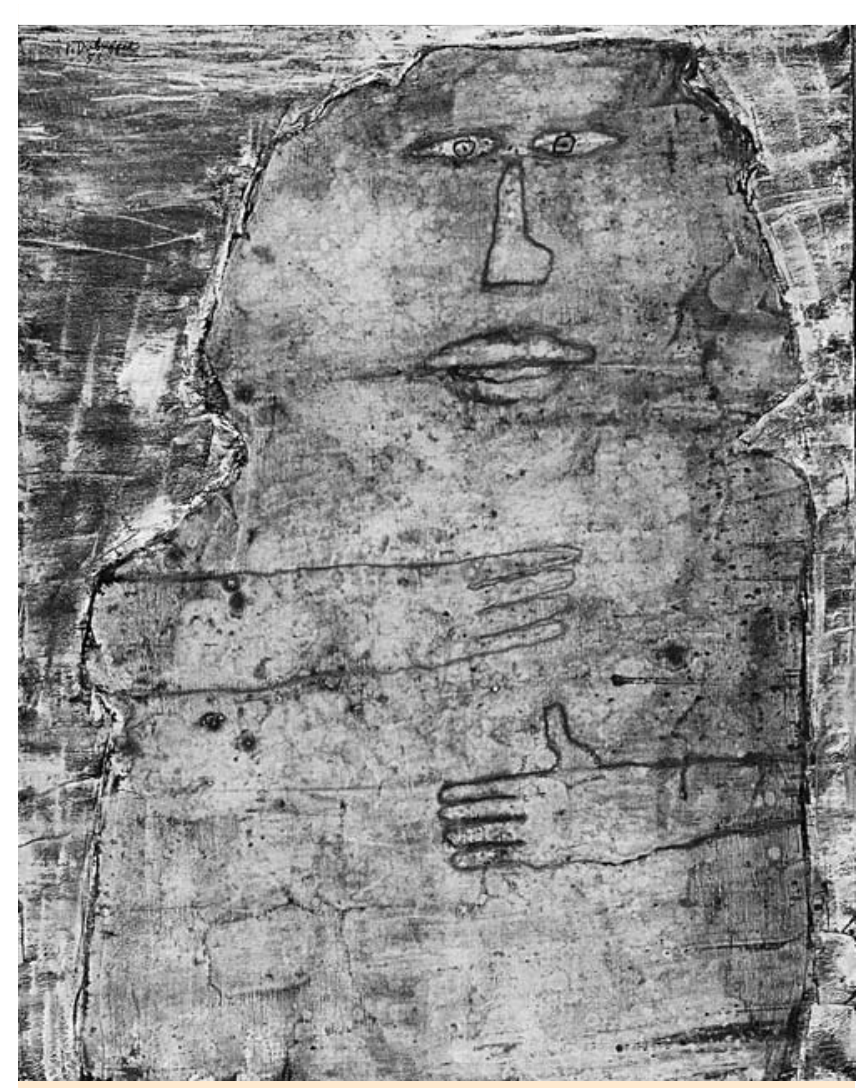

Abb. 1 Jean Dubuffet, L'Albinos, 1958, Öl/Leinwand (Wilhelm-Hack-Museum Ludwigshafen, ( $)$ VG Bild-Kunst, Bonn 2011).

François Morellet Zufällige Verteilung auf schwarzem Grund und Zufällige Verteilung auf weißem Grund fand bei der Betrachtung der Sammlung besondere Aufmerksamkeit [3]. Die farbigen Quadrate in netzartiger Struktur präsentieren sich dem Betrachter bedingt durch ihren hellen oder dunklen Hintergrund in jeweils unterschiedlicher farblicher Wertigkeit. Die exakt gleiche Farbe verändert mit Blick auf einen weißen oder aber schwarzen Bildgrund ihre Brillanz und ihre Wirkung, und die exakt gleiche quadratische Form scheint in ihrer Textur verändert. Die Wahrnehmung der Farben ist abhängig vom Hintergrund - eine Analogie zu Erkrankungen auf dunkler oder heller Haut.

Aktives Betrachten, also das buchstäbliche Abtasten der Leinwand mit den Augen, ist in besonderer Weise bei den sogenannten abstrakten oder lyrischen Expressionisten notwendig. Der amerikanische Künstler Jackson Pollock ist mit seinen dripping paintings berühmt geworden, die die Malerei als Aktion zelebriert. Pollock ließ Farbe aus durchlöcherten Farbtöpfen ganzflächig auf die am Boden liegende Leinwand tröpfeln und fließen, er schüttete, spritzte und spachtelte sie derart, dass dynamische Strukturen, zarte Gespinste und Linien und vollendete Rhythmen entstanden. Diese Praxis hat dem Künstler noch zu Lebzeiten den Spitznamen „Jack, the dripper“ eingebracht. In Analogie zur Haut entstehen so Oberflächenschichten und feinste Reliefs, bestehend aus Farbfasern, welche die Leinwand überziehen und sich zu einem Gesamtbild formen.

\section{Künstlerische Metapher „Haut" \\ $\nabla$}

Symbole und Metaphern verdeutlichen das gesellschaftlich gesponnene Bedeutungsgewebe von Kultur in all seiner Komplexität. Auch die Haut ist ein zentrales Sujet in Kunst und Literatur [2]. Aus der Haut fahren, eine Gänsehaut bekommen, die eigene Haut retten, eine dünne Haut haben, unter die Haut gehen, Haut und Knochen sein, sind nur einige einer breiten Palette von Redewendungen. Haut hat viele Künstler und Autoren als Grenzfläche zwischen Innen und Außen, als sensitive Schutzhülle und als Organ mit Schmerzsymptomen inspiriert. Im Surrealismus taucht der Begriff der Oberfläche eines Gemäldes als „Haut der Malerei“ auf, eine zentrale Metapher für die Aufhebung der Grenze von Kunst und Leben - eine Haut, die die Malerei gleichsam erst erschafft [7]. Findet sich nackte Haut als verführerisches Merkmal des Menschen allenthalben in den großen Meisterwerken der Kunst, so wird das größte menschliche Organ in Werken des 20. Jahrhunderts zur gefährdeten Hülle des fragilen Individuums. Leinwände werden von Künstlern zum Ziel der Öffnung perforiert und durchschlitzt, gewissermaßen verletzt. Die Suche nach Identität und der wandelbaren Konstitution des Selbst findet sich poetisch sowohl im Thema des Eingenähtseins in Haut als auch im Sich-Schälen, im Hautablegen und im Anlegen einer anderen Haut. Der Topos der Häutung kann als schmerzhafter Selbstverlust oder aber als Vorgang der Reinigung und der Metamorphose verstanden werden. Darüber hinaus wird helle und dunkle Haut in künstlerischen Statements als visuelle Kategorie der Andersheit thematisiert.

\section{Diagnostischer Blick und leibhaftige Erfahrung $\nabla$}

Wie der Dermatologe bei der Betrachtung von Haut, stellt sich der Kunsthistoriker bei der Kunstbetrachtung die Frage, was man sieht und wie man das, was man sieht, sprachlich ausdrücken kann. Mit seinem kunsthistorischen Rucksack ordnet der Kurator seinen Gegenstand in einen größeren Kontext ein und erkennt gesellschaftliche Symptome einer Epoche, einer bestimmten Zeit. Gibt es in der Medizin eine Terminologie der Krankheiten, so hat die klassische Kunstgeschichte mit ihrer ikonografisch-ikonologischen Methode eine Terminologie der Kunstbegriffe hervorgebracht. Ästhetik (aísthesis) bezeichnet ganz allgemein die Lehre von der sinnlichen Wahrnehmung mit ihrem breiten Spektrum formaler, intellektueller und emotionaler Dimensionen. Die komplexe Frage nach der Subjektivität und Objektivität bei der Kunstbetrachtung hat das Fach - vereinfacht reduziert - mit der Unterscheidung zwischen Subjekt- und Objektästhetik beantwortet. So fokussiert die Objektästhetik das Werk als technisches und historisches Gebilde, während die Subjektästhetik Wirkung, Geschmack und Individuum analysiert. Neben den physiognomischen Aspekten des Sehens, gewissermaßen des diagnostischen Blicks, ist Kunstbetrachtung immer auch leibhaftige Erfahrung. Eine Reproduktion ersetzt nicht den „Körper“ des Kunstwerks, dem man als Betrachter seinen eigenen Körper mit seinem Fühlen, seiner synästhetischen Auffassungsgabe und seiner Sensibilität für die Wirkung von Material - Leichtigkeit, Schwere, Fragilität, Monumentalität - entgegensetzt. 


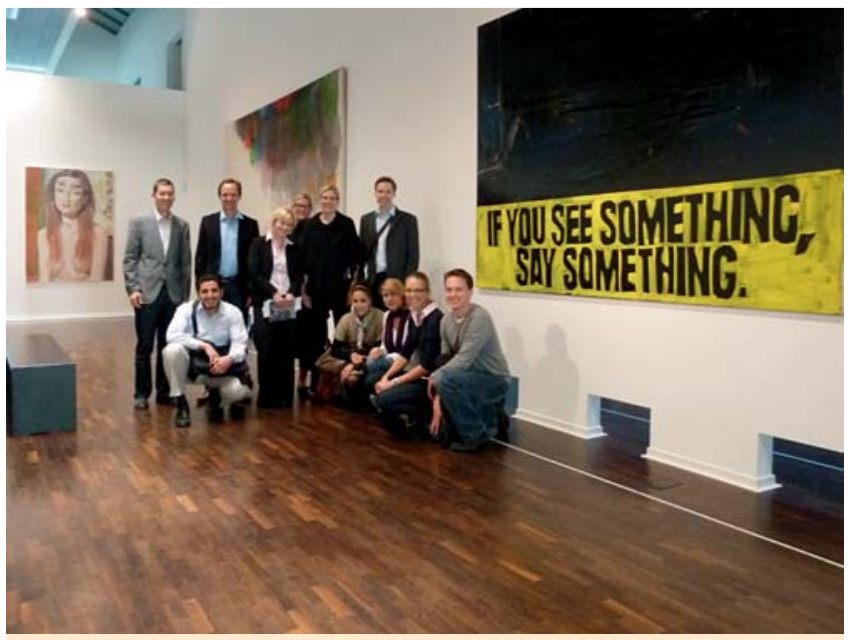

Abb. 2 Ärzte der Hautklinik Ludwigshafen zu Gast im Wilhelm-Hack-Museum.

\section{Fazit}

$\nabla$

Ist neben der ästhetischen und intellektuellen Dimension eines Kunstwerks der emotionale Zugang bei der Kunstbetrachtung zentral, so dienen Gefühle bei der Erhebung eines Hautbefundes allenfalls in mnemotechnischem Kontext zur Erfassung der Diagnose. Die regelmäßige persönliche Auseinandersetzung mit der Kunst und die Schulung des Blicks durch gezielte Betrachtung kann die Perzeption von Formen, Farben und Kontrasten verbessern. Exemplarisch kann hierfür nochmals ein Kunstwerk herangezogen werden, das Haut zu seinem zentralen Thema macht der Albino von Jean Dubuffet ( $\bullet$ Abb. 1).

Dubuffets Programm einer „rohen Kunst“, die sich gerade nicht in konventionellen und eingeübten Formen ausdrücken sollte, mag sinnbildlich für eine unkonventionelle dermatologische Weiterbildung stehen: Warum nicht den Blick schärfen durch Besuche im Museum, denn auch hier liegt das Gute wie so oft unerwartet nah, gerade in Ludwigshafen ( $\bullet$ Abb. 2).

\section{Danksagung}

Herrn Professor Dippel für seine ideelle und finanzielle Förderung dieser unkonventionellen Fortbildungsmaßnahmen.

\section{Abstract}

\section{New Paths in Dermatologic Education \\ Part 2: Hospital Meets Art: Vision-Training for Dermatologists - Physicians of the Skin Hospital Visit the Wilhelm-Hack-Museum in Ludwigshafen}

Dermatology demands from its board certified proponents to recognize structures and connections, everyone can see on the skin, but only the trained eye will recognize. Therefore in 2010, on the initiative of the author, the physicians of the Skin Hospital in Ludwigshafen were invited in addition to several conventional educational meetings to train their senses in a most exceptional way.

Because visual aspects in dermatology today fortunately surpass other aspects, the second special event took all physicians of the Skin Hospital to the Wilhelm-Hack-Museum in Ludwigshafen. Under the title „vision-training for dermatologists“ Dr. Judith Elisabeth Weiss, then curator of the museum, lead through the collection where important international works of $20^{\text {th }}$ century art are presented.

\section{Literatur}

1 Bardes CL, Gillers D, Herman AE. Learning to look: developing clinical observational skills at an art museum. Med Educ 2001; 35: $1157-$ 1161

2 Benthien C. Haut. Literaturgeschichte - Körperbilder - Grenzdiskurse. Frankfurt: 1999

3 Gassen RW. Kunst des 20. Jahrhunderts, Wilhelm-Hack-Museum. Ludwigshafen am Rhein: 1999

$4 \mathrm{Kerl} \mathrm{H}$, Wolf IH, Keil Ket al. Freude an der Morphologie: Dermatopathologie und Kunst. J Deutsch Dermatol Ges 2010; 8: 917-919

5 Naghshineh S, Hafler JP, Miller AR et al. Formal art observation training improves medical students' visual diagnostic skills. J Gen Intern Med 2008; 23: 991 - 997

6 Shapiro J, Rucker L, Beck J. Training the clinical eye and mind: using the arts to develop medical students' observational and pattern recognition skills. Med Educ 2006; 40: 263 - 268

7 Tériade. La peau de la peinture. in: MINOTAURE: revue artistique et littéraire, No. 7; 1935 